\title{
Reduction in postsurgical adhesion formation after cardiac surgery by application of $\mathrm{N}, \mathrm{O}$-carboxymethyl chitosan
}

\author{
Juan Zhou, MD, PhD, ${ }^{\text {a,b }}$ J. Michael Lee, PhD, ${ }^{\text {a,c }}$ Patricia Jiang, BSc, ${ }^{\mathrm{b}}$ Susan Henderson, BSc, ${ }^{\mathrm{e}}$ and \\ Timothy D. G. Lee, $\mathrm{PhD}^{\mathrm{a}, \mathrm{b}, \mathrm{d}}$
}

\begin{abstract}
Objective: The study objectives were to assess the efficacy of N,O carboxymethyl chitosan film in reducing postsurgical adhesion in a rabbit cardiac injury model and to confirm the efficacy of N,O carboxymethyl chitosan gel and film in reducing postsurgical adhesion formation in a pig cardiac injury model.
\end{abstract}

\begin{abstract}
Methods: (1) Rabbit cardiac injury model: Cardiac injury was generated by abrading the anterior surface of the heart and desiccation with oxygen. N,O carboxymethyl chitosan solution and film were administered to the injured surface. (2) Pig cardiac injury model: Cardiac injury was generated as described above. N,O carboxymethyl chitosan solution and gel (or film) were administered to the injured surface. The severity and area of adhesion between the heart and the sternum were evaluated at 14 days postcardiac surgery.
\end{abstract}

Results: (1) Rabbits treated with N,O carboxymethyl chitosan film plus solution showed significantly reduced severity and area of adhesion formation. (2) Both N,O carboxymethyl chitosan gel plus solution and N,O carboxymethyl chitosan film plus solution significantly reduced adhesion formation in the pig model.

Conclusions: Application of N,O carboxymethyl chitosan products significantly reduces severity of postsurgical adhesion formation after cardiac surgery in the rabbit and pig models. N,O carboxymethyl chitosan products may act as a biophysical barrier. (J Thorac Cardiovasc Surg 2010;140:801-6)

Postsurgical adhesion formation is a common complication of cardiac surgery. Adhesion formation not only is troublesome for secondary cardiac surgery, involving increased risks of heart injury and severe hemorrhage, but also may obliterate the pericardial space and constrict heart function. ${ }^{1}$ A product that can prevent adhesion formation after cardiac surgery would be of great benefit. A number of approaches to reduce or prevent adhesion formation have been examined in animal models, and some therapies have reached clinical trials and the marketplace. A common feature in a number of these interventions has been an attempt to establish a physical barrier between injured surfaces by the application of films or gels. ${ }^{2-4}$ Problems have arisen limiting the ease of use of many of these products in the clinical setting. For example, polytetrafluoroethylene membranes have been demonstrated to effectively reduce cardiac adhesion formation in clinical

\footnotetext{
From the Departments of Surgery, ${ }^{\mathrm{a}}$ Microbiology and Immunology, ${ }^{\mathrm{b}}$ School of Biomedical Engineering, ${ }^{\mathrm{c}}$ and Pathology, ${ }^{\mathrm{d}}$ Faculty of Medicine, Dalhousie University, Halifax, Nova Scotia; and Kytogenics Pharmaceuticals Ltd, ${ }^{\mathrm{e}}$ Dartmouth, Nova Scotia, Canada.

This work was supported in full by Kytogenics Pharmaceuticals Ltd.

Disclosures: S. Henderson is a shareholder in Kytogenics Pharmaceuticals Ltd.

J. Zhou, M. Lee, P. Jiang, and T. Lee have no financial interest in Kytogenics Ltd. Received for publication July 24, 2009; revisions received Oct 17, 2009; accepted for publication Nov 14, 2009; available ahead of print Feb 22, 2010.

Address for reprints: Timothy D. G. Lee, PhD, Sir Charles Tupper Building, 5850 College Street, Dalhousie University, Halifax, NS, Canada, B3H 4 H7 (E-mail: tim.lee@dal.ca).

$0022-5223 / \$ 36.00$

Copyright $(c) 2010$ by The American Association for Thoracic Surgery

doi:10.1016/j.jtcvs.2009.11.030
}

trials. Unfortunately, these membranes persist in the chest cavity and become encapsulated, leading to complications. ${ }^{5}$ A more effective product in the long term would exhibit the barrier effect of the polytetrafluoroethylene membranes but be completely biodegradable over a limited time period.

A chitosan derivative, N,O-carboxymethyl chitosan (NOCC), has shown significant promise as an anti-adhesion agent. NOCC can be formulated as a solution, gel, or film. In all of these forms, NOCC is biodegradable within 5 days. We have provided a significant amount of evidence to date demonstrating that NOCC gel plus NOCC solution limits adhesion formation and adhesion reformation in animal abdominal injury models. ${ }^{6-8}$ In addition to the preclinical evidence, NOCC gel plus NOCC solution has been shown to reduce adhesion reformation in stage I and II clinical trials. ${ }^{9}$ We recently demonstrated that application of NOCC gel plus NOCC solution significantly reduces adhesion formation after cardiac surgery in a rabbit cardiac injury model. ${ }^{10}$ The reduction of adhesions was suggested to be due to the prevention of fibroblast and macrophage deposition on the injured sites, resulting in reduced adhesion formation. ${ }^{10}$

The present study assesses the effect of NOCC film, a new NOCC formulation used along with NOCC solution, on adhesion formation after cardiac surgery in the rabbit cardiac injury model that we have used previously. ${ }^{10}$ Moreover, we confirm the positive effects of NOCC products in a large animal cardiac surgery model. We demonstrate that application of NOCC products significantly inhibits postsurgical adhesion formation after cardiac surgery in the pig. 


\section{Abbreviation and Acronym \\ $\mathrm{NOCC}=\mathrm{N}, \mathrm{O}$-carboxymethyl chitosan}

\section{MATERIALS AND METHODS Animals}

Female New Zealand White rabbits $(2.5-3.0 \mathrm{~kg})$ were purchased from Charles River Laboratories (Wilmington, Mass). Male Hampshire Duroc Cross pigs (30-35 kg) were purchased from Mapleline Farms. All the animals were maintained in the Carleton Animal Care Facility of the Faculty of Medicine, Dalhousie University, and provided with food and water ad libitum. All animal experimentation was undertaken in compliance with the guidelines of the Canadian Council of Animal Care.

\section{N,O-Carboxymethyl Chitosan Products}

Three NOCC products were provided by Kytogenics Pharmaceuticals Ltd (Dartmouth, Nova Scotia, Canada). They included a non-cross-linked $2 \%$ NOCC solution, a 1\% NOCC gel cross-linked with gyloxal, and a NOCC film that was made from a mixture of NOCC solution and glycerol, and then cross-linked with glyoxal. Both gel and solution were provided sterile and used in an aseptic manner in the cardiac injury model described below. The NOCC film was cut into $2-\mathrm{cm}$ diameter circles and soaked in $70 \%$ ethanol overnight in a sterile Petri dish the night before use in rabbit model. The film was washed under aseptic conditions with sterile saline and then soaked for 1 hour in 2 changes of sterile saline to remove all of the ethanol. This presoaked film was kept in sterile saline ready for use. In the pig cardiac injury model, the NOCC film was trimmed into appropriate shape and size (oval, $\sim 2 \times 4 \mathrm{~cm}$ ) of injury area, sprayed with $70 \%$ ethanol, and soaked in saline for 2 minutes immediately before application.

\section{Cardiac Injury Model in Rabbits}

All surgeries were performed by the principal investigator (T.L.) assisted by the project director (J.Z.). Both were blinded to the treatment to be applied after the surgery. Surgery was performed in the fully equipped animal operating room (Canadian Council of Animal Care approved) in the Carleton Animal Care Facility. Cardiac activity was monitored with an electrocardiograph throughout the entire surgery. Anesthesia was performed and monitored by trained veterinary surgical nurses.

Rabbits were anesthetized with ketamine $(50 \mathrm{mg} / \mathrm{kg})$ and xylazine $(5 \mathrm{mg} /$ $\mathrm{kg}$ ) intramuscular injection for induction, followed by endotracheal intubation and mechanical ventilation and maintained with isoflurane $(3 \%)$. After preparation for aseptic surgery, a midline incision of skin and muscles was made with a scalpel and electrocautery, respectively. Bleeding was controlled with electrocautery. The thorax was opened via a median sternotomy using straight surgical scissors, and a sternal retractor was placed to obtain adequate exposure. Great care was taken to not create a pneumothorax and deflate the lung. The anterior pericardium was opened at approximately 4 to $5 \mathrm{~cm}$ in length to expose the anterior surface of the heart. The anterior surface of the heart $(\sim 2 \times 1 \mathrm{~cm})$ was abraded for 5 minutes with gauze (to develop punctuate bleeding) and desiccated twice (30 seconds each time) with oxygen at a flow rate of $5 \mathrm{~L} / \mathrm{min}$. After the injury, animals were either (1) treated with NOCC products by placing a presoaked NOCC film (as described above) on the injured surface of the heart and $2 \mathrm{~mL}$ NOCC solution over and around the film or (2) not treated with any NOCC products (as a control group). After treatment, leaving the pericardium open, the sternum was closed with interrupted 2-O Dexon sutures. The muscle and skin were closed in layers with 3-0 Dexon and staples, respectively. Air was sucked from the chest via a tube placed just before closure of the sternum. This prevented tamponade and provided extra insurance against pneumothorax. All animals received analgesic (morphine $2.0 \mathrm{mg} / \mathrm{kg}$ intravenously) and antibi- otics (Baytril [Bayer AG, Leverkusen, Germany], $5 \mathrm{mg} / \mathrm{kg}$, by subcutaneous injection) at the time of surgery, and 2 additional doses of analgesic (buprenorphine, $0.05 \mathrm{mg} / \mathrm{kg}$ by subcutaneous injection) at 12 and 24 hours postsurgery. Animals recovered in heated cages, with frequent observation, and received food and water ad libitum.

At 14 days after the surgery, rabbits were sacrificed by lethal injection of sodium pentobarbital $(340 \mathrm{mg} / \mathrm{mL})$ for assessment of poststernal adhesion formation (adhesions between the heart and the sternum). The anterior chest, including the ribs, sternum, and heart adherent to the sternum, was removed from rabbits. The posterior pericardium was opened, and adhesions between the heart and the sternum were outlined with India ink. The severity of adhesions was evaluated by the tensile force required to separate the heart from the sternum and the manner in which the tissue could be separated on a 5-point scale: grade 0 , no adhesions; grade 1, very weak adhesions that were easy to tear apart by hand; grade 2 , weak adhesions that can be dissected with fingers; grade 3, moderately strong adhesions that require partial dissection with scissors; grade 4, strong adhesions that require dissection with scissors; and grade 5, very strong adhesions that need to be dissected with scissors. After removal of the heart from the sternum, the areas of adhesion could be visualized by the Indian ink outline. These were photographed, and the areas of adhesion indicated by the markers were evaluated by a computerized digital image analysis.

All animals were randomly assigned to the treatment or control group before surgery. The surgeon performing the operation was blinded to the assignment. All animals were ear tattooed by animal care personnel immediately before the operation. After the cardiac injury was complete, the NOCC film and solution were applied by a technician who was aware of the treatment assignment and tattoo number but not otherwise involved in the operation. On the day of sacrifice, all animals were randomly presented for evaluation by the animal care staff. Evaluation of adhesions was performed by the operating surgeon. The surgical assistant noted the tattoo number and the evaluation. After all the animals were evaluated, adhesion data, including the severity and area of adhesion, were provided to the treatment technician, who then matched the tattoo numbers recorded with the treatment applied and the adhesion data recorded.

\section{Cardiac Injury Model in Pigs}

The pigs were anesthetized with ketamine $(50 \mathrm{mg} / \mathrm{kg})$ and xylazine $(5 \mathrm{mg} /$ $\mathrm{kg}$ ) intramuscular injection for induction, followed by endotracheal intubation and mechanical ventilation and maintained with isoflurane $(3 \%)$ throughout the entire surgery. After preparation for aseptic surgery, a midline incision of skin and muscles was made with a scalpel and electrocautery, respectively. Bleeding was controlled with electrocautery. Before medial sternotomy, $2 \%$ lidocaine $(1 \mathrm{mg} / \mathrm{kg}$ bolus dose and $50 \mu \mathrm{g} / \mathrm{kg} / \mathrm{min}$ constant drip throughout the following surgery) was given intravenously to prevent ventricular arrhythmias. The thorax was opened via a medial sternotomy using a high-speed oscillating saw (Stryker Instrument, Portage, MI). The connective tissue between the sternum and the pericardium was cleared with blunt dissection, and a sternal retractor was placed to obtain adequate exposure. The anterior pericardium was opened longitudinally to expose the anterior surface of the heart, which was then lightly abraded 50 times with sandpaper (grade 120) to develop punctuate hemorrhage. The heart was then desiccated with oxygen for 1 minute at a flow rate of $10 \mathrm{~L} / \mathrm{min}$. The heart was then abraded another 10 times and desiccated for 1 minute. During the abrasion period, cardiac activity was closely monitored via electrocardiograph. Every 10 times, abrasion was stopped to allow the heart to recover from premature ventricular contractions. After abrasion and desiccation the hearts were then treated (1) with $50 \mathrm{~mL}$ NOCC gel and $25 \mathrm{~mL}$ solution; (2) with NOCC film and $25 \mathrm{~mL}$ solution; or (3) not treated. In the NOCC-treated animals, NOCC gel or film was placed on the injured surface of the heart and NOCC solution was applied around the gel or film and on the top of the film. By leaving the pericardium open, the sternum was closed with 6 interrupted stainless-steel sutures. The muscle and subcutaneous fascia were closed in layers with 0 0 Dexon suture, and the skin was closed with staples. All animals received 
analgesic (morphine $2.0 \mathrm{mg} / \mathrm{kg}$ intravenously) and antibiotics (Baytril, $5 \mathrm{mg}$ / $\mathrm{kg}$, by subcutaneous injection) at the time of surgery and postsurgical analgesic (buprenorphine $0.05 \mathrm{mg} / \mathrm{kg}$ subcutaneous patch). Animals recovered in individual cages, with frequent observation, and received food and water ad libitum.

At $14 \pm 1$ days after surgery, animals were sacrificed by lethal injection of sodium pentobarbital. The anterior chest, including the ribs, sternum, and heart adherent to the sternum, was removed from the pig for analysis of poststernal adhesion formation (adhesions between the heart and the sternum). The pericardium was dissected from the heart. The adhesion between the heart and the sternum was outlined with India ink. The heart and the sternum marked with India ink were photographed, and the areas of adhesion indicated by the markers were evaluated by a computerized digital image analysis.

The severity of adhesions was evaluated in terms of the peak tensile force required to pull the heart off the sternum. On an Instron $8821 \mathrm{~S}$ electromechanical testing system (Instron Corporation, Norwood, Mass), the heart was supported in a rope sling attached to a $50-\mathrm{kg}$ load cell, and the sternum was held in a clamp grip via stainless-steel surgical wires. With the sample well hydrated with Hanks' solution, the heart and sternum were separated at a rate of $10 \mathrm{~mm} / \mathrm{min}$ such that the adhesion was subjected to tensile force nearly normal to the plane of the sternum. Force/time data were recorded to computer using software written under National Instruments Corp's LabVIEW platform (Austin, Tex). The peak force required to separate the heart from the sternum was obtained from this dataset. All procedures and evaluations were performed in a blinded fashion as described above.

\section{Statistical Analysis}

Given that the data showed a nonparametric distribution, a Wilcoxon rank-sum test was used throughout and data are presented as group medians rather than mean \pm standard deviation.

\section{RESULTS}

\section{Application of N,O-Carboxymethyl Chitosan Film Significantly Reduced Adhesion Formation After Rabbit Cardiac Surgery}

All 20 rabbits that underwent cardiac surgery survived the surgery and were maintained without morbidity for 14 days until sacrifice. In this experiment, we examined adhesions between the epicardium and the sternum. In all but 1 animal, the cut edge of the pericardium and the damaged epicardium of the heart were adherent to the posterior surface of the sternum. However, the severity of adhesions in NOCC film and solution-treated animals was significantly $(P<.05)$ reduced compared with that in control animals (Table 1). Nine of the 10 animals in the control group had strong adhesions of grades 4 and 5 . These adhesions required sharp dissection to separate the heart from the sternum. In contrast, in the NOCC film and solution-treated group, all the animals showed weak and loose adhesions (grades 1 and 2) that were easily separated with fingers.

The area of adhesions between the heart and the sternum was obtained through computerized image analysis of the area outlined by the India ink. The results (Table 1) demonstrate that there was a significant $(P<.05)$ reduction in the median adhesion area in NOCC-treated animals when compared with nontreated control animals.

A cumulative adhesion score for each animal, calculated by multiplying the adhesion grade and adhesion area, was
TABLE 1. Postsurgical adhesion formation after cardiac surgery in rabbits after $\mathrm{N}, \mathrm{O}$-carboxymethyl chitosan film and solution treated (N,O-carboxymethyl chitosan) or not treated (control)

\begin{tabular}{lcccccccc}
\hline & \multicolumn{2}{c}{ Severity } & & \multicolumn{2}{c}{ Area $\left(\mathbf{c m}^{2}\right)$} & & \multicolumn{2}{c}{ Cumulative score } \\
\cline { 2 - 3 } Animal no. & NOCC & Control & & NOCC & Control & & NOCC & Control \\
\hline 1 & 2 & 3 & & 4.7 & 4.4 & & 9.4 & 13.5 \\
2 & 0 & 5 & & 0.0 & 6.1 & & 0.0 & 30.3 \\
3 & 1 & 5 & & 1.1 & 3.8 & & 1.1 & 19.2 \\
4 & 1 & 5 & & 2.9 & 3.1 & & 2.9 & 15.6 \\
5 & 1 & 4 & & 0.3 & 2.3 & & 0.3 & 9.3 \\
6 & 1 & 4 & & 0.4 & 2.4 & & 0.4 & 9.4 \\
7 & 2 & 4 & & 2.4 & 3.1 & & 4.7 & 12.4 \\
8 & 2 & 5 & & 0.3 & 1.8 & & 0.6 & 9.2 \\
9 & 1 & 4 & & 0.7 & 2.5 & & 0.7 & 10.0 \\
10 & 2 & 4 & & 0.5 & 2.2 & & 1.0 & 8.7 \\
Median & $1 *$ & 4 & & $0.6 *$ & 2.8 & & $0.9 \dagger$ & 11.2 \\
\hline
\end{tabular}

NOCC, N,O-carboxymethyl chitosan. Severity of adhesion is measured by grades 1 to 5. Area of adhesions is expressed as centimeters squared. Cumulative adhesion score is established by multiplying the severity of adhesions with the area of adhesions. Significantly different $(* P<.05 ; \nmid P<.01$, Wilcoxon rank-sum test) from control.

used to evaluate the overall adhesion formation. Statistical analysis confirmed a significantly lower cumulative score of the NOCC-treated group $(P<.01)$ when compared with the nontreated control group (Table 1).

\section{Application of N,O-Carboxymethyl Chitosan Gel or Film Significantly Reduced Adhesion Severity After Pig Cardiac Surgery}

Twenty-one pigs underwent cardiac surgery. At the time of initial surgery, 1 pig showed an abnormal heart structure with severe adhesions of the heart to the pericardium. This animal was presumed to have had pericarditis in the past (of unknown cause) and was sacrificed during surgery and excluded from the experiment. All other pigs showed normal cardiac morphology and survived the surgery. At $14 \pm 1$ days postsurgery, all 20 pigs were sacrificed and adhesion between the pericardium and the sternum was examined. All but 1 of the animals developed adhesions between the anterior surface of the heart and the posterior surface of the sternum. The severity of adhesions was significantly weaker in NOCC gel plus solution-treated animals than in nontreated animals.

Under mechanical testing, the first visible failure occurred variably around the margin of the adhesion or was not visible at all when the peak force was recorded. This suggests that the adhesion failed in near-uniform tension rather than peeling away. As shown in Table 2, the severity of adhesions, measured by the force required to break the adhesion in the control nontreated group, ranged from 3.6 to $15.4 \mathrm{~kg}$ with a median of $5.75 \mathrm{~kg}$. In contrast, the severity of adhesions in the NOCC gel plus solution-treated group was significantly $(P<.05)$ weaker (range, 0-4.1 kg; median, $2.2 \mathrm{~kg}$ ). One of the NOCC geltreated animals did not show any adhesions between the heart 
TABLE 2. Severity of adhesions between heart and sternum after cardiac surgery in pigs after $\mathrm{N}, \mathrm{O}$-carboxymethyl chitosan gel and solution treated (N,O-carboxymethyl chitosan gel/sol) or N,O-carboxymethyl chitosan film and solution treated (N,O-carboxymethyl chitosan film/sol) or not treated (control)

\begin{tabular}{lccc}
\hline Animal no. & NOCC gel/sol & NOCC film/sol & Control \\
\hline 1 & 4.1 & 3.3 & 4.9 \\
2 & 0 & 2.8 & 15.4 \\
3 & 0.9 & 6.6 & 3.6 \\
4 & 4.1 & 5.4 & 6.6 \\
5 & 2.2 & 2.4 & 3.6 \\
6 & 2.7 & 1.9 & 10.2 \\
7 & 0.3 & 0.3 & \\
Median & $2.2^{*}$ & $2.8^{*}$ & 5.8 \\
\hline
\end{tabular}

NOCC, N,O-carboxymethyl chitosan. The severity of adhesions is expressed at the peak tensile force (kilograms) required to separate the heart from the sternum in a pull-to-failure test. ${ }^{*}$ Significantly different $(P<.05$, Wilcoxon rank-sum test $)$ from control.

and the sternum. Likewise, the severity of adhesions was significantly reduced in NOCC film plus solution-treated animals when compared with the nontreated animals (Table 2; $P<$ $.05)$. The severity of adhesions in the NOCC film plus solution ranged from 0.3 to $6.6 \mathrm{~kg}$ and a median of $2.8 \mathrm{~kg}$. As noted above, the range in the nontreated control was 3.6 to $15.4 \mathrm{~kg}$ with a median of $5.75 \mathrm{~kg}$.

\section{Application of N,O-Carboxymethyl Chitosan Gel or Film Significantly Reduced Adhesion Area After Pig Cardiac Surgery}

The area of adhesions between the heart and the sternum obtained through computerized image analysis is shown in Table 3. There is a significant $(P<.05)$ reduction in the adhesion area in NOCC gel plus solution-treated animals when compared with nontreated control animals. Control adhesions ranged from 11.4 to $27.2 \mathrm{~cm}^{2}$ with a median of 13.8 $\mathrm{cm}^{2}$. In contrast, the NOCC gel plus solution group showed a range between 0 and $13.3 \mathrm{~cm}^{2}$ with a median of $7.5 \mathrm{~cm}^{2}$. Likewise, the area of adhesions was significantly reduced in NOCC film plus solution-treated animals when compared with the nontreated animals (Table $3, P<.05$ ). The area of adhesions in the NOCC film plus solution ranged from 0.8 to $20.16 \mathrm{~cm}^{2}$ with a median of $8.72 \mathrm{~cm}^{2}$.

\section{Application of N,O-Carboxymethyl Chitosan Gel or Film Significantly Reduced Cumulative Adhesion Score After Pig Cardiac Surgery}

A cumulative adhesion score calculated by multiplying the adhesion severity and adhesion area is presented in Table 4. Statistical analysis confirmed that the cumulative scores of the NOCC gel plus solution-treated group and the NOCC film plus solution-treated group were both significantly $(P<.01)$ lower than that of the nontreated control group.

These data confirm that NOCC gel and solution and NOCC film plus solution applied on the injured surfaces both reduce
TABLE 3. Area of adhesions after cardiac surgery in pigs after N,Ocarboxymethyl chitosan gel and solution treated (N,O-carboxymethyl chitosan gel/sol) or N,O-carboxymethyl chitosan film and solution treated (N,O-carboxymethyl chitosan film/sol) or not treated (control)

\begin{tabular}{lccc}
\hline Animal no. & NOCC gel/sol & NOCC film/sol & Control \\
\hline 1 & 8.3 & 8.7 & 12.0 \\
2 & 0.0 & 14.6 & 14.0 \\
3 & 1.7 & 14.6 & 11.4 \\
4 & 10.0 & 20.2 & 20.6 \\
5 & 13.3 & 1.6 & 13.5 \\
6 & 7.5 & 0.8 & 27.2 \\
7 & 1.4 & 5.5 & \\
Median & $7.5^{*}$ & $8.7 *$ & 13.8 \\
\hline NOCC, N,O-carboxymethyl chitosan. The area of adhesions is expressed as centime- \\
ters squared. *Significantly different $(P<.05$, Wilcoxon rank-sum test) from control.
\end{tabular}

adhesion formation after cardiac surgery in a pig model. There was no significant difference between the 2 treated groups, indicating that both the gel and the film are equally effective at reducing adhesion formation $(P>.05)$.

\section{DISCUSSION}

NOCC has been shown to significantly prevent adhesion formation after abdominal and gynecologic surgery in animal models. ${ }^{6-8}$ We recently demonstrated that application of NOCC gel plus NOCC solution on the surgically injured heart surface significantly reduces postsurgical adhesion formation in a rabbit cardiac injury model. ${ }^{10}$ In the present study, using the same rabbit cardiac injury model, we assessed the ability of NOCC film to prevent adhesion formation after cardiac surgery. In addition, using a more clinically relevant pig cardiac injury model, we confirmed the effect of NOCC gel and NOCC film on adhesion formation. The data reported indicate that application of NOCC gel or NOCC film combined with application of NOCC solution significantly reduces the extent of adhesion formation after cardiac surgery. In our experiments, we evaluated adhesion formation at 2 weeks postsurgery with NOCC products. In our previous studies with NOCC products, adhesion formation at 2 weeks and 3 months after cardiac surgery showed identical results. ${ }^{10}$

It has been demonstrated that the presence of a physical barrier between injured tissue surfaces during an early stage of healing (16-36 hours postsurgery) is critical for preventing adhesion formation. ${ }^{11-13}$ We have found that NOCC film maintains its structural integrity in the rat thoracic cavity for 5 days and degrades after 7 days of insertion (T.D.G. Lee, $\mathrm{PhD}$, unpublished data, 2004). NOCC gel has been demonstrated to entirely degrade within 5 days of delivery. In the present study, no evidence of the NOCC film was seen at harvest, 2 weeks after treatment. The results from both rabbit and pig cardiac injury models demonstrate that NOCC film plus solution significantly reduced the severity and area of retrosternal adhesion formation after cardiac surgery. 
TABLE 4. Cumulative score of adhesions after cardiac surgery in pigs after N,O-carboxymethyl chitosan gel and solution treated (N,O-carboxymethyl chitosan gel/sol) or N,O-carboxymethyl chitosan film and solution treated (N,O-carboxymethyl chitosan film/ sol) or not treated (control)

\begin{tabular}{lccr}
\hline Animal no. & NOCC gel/sol & NOCC film/sol & Control \\
\hline 1 & 34.1 & 28.8 & 58.8 \\
2 & 0.0 & 41.0 & 215.6 \\
3 & 1.5 & 96.1 & 41.2 \\
4 & 41.2 & 108.9 & 136.2 \\
5 & 29.3 & 3.9 & 48.7 \\
6 & 20.3 & 1.6 & 277.4 \\
7 & 0.4 & 1.6 & \\
Median & $20.3 \dagger$ & $28.8^{*}$ & 97.5 \\
\hline
\end{tabular}

NOCC, N,O-carboxymethyl chitosan. Cumulative score is established by multiplying the severity of adhesions with the area of adhesions. Significantly different $\left({ }^{*} P<.05\right.$; $\dagger P<.01$ Wilcoxon rank-sum test) from control.

Similar results were obtained when NOCC gel plus solution was applied.

Evidence to date suggests that NOCC gel or NOCC film applied on the site of injury forms a direct physical barrier at the injury site, whereas NOCC solution prevents adhesion formation initiated by more general minor abrasion during surgery. By using an abdominal injury model, we previously demonstrated that application of NOCC gel to the site of injury is critical to blocking adhesion formation. ${ }^{8}$ Application of NOCC solution alone did not prevent adhesion formation at the injury site but did significantly reduce more minor adhesions in areas closely associated with the surgical site and likely to experience minor abrasion damage. NOCC gel alone reduced adhesions at the site of surgery but did not reduce adhesion formation at associated sites. Because NOCC film is absorbable and easy to handle, the application of NOCC film with NOCC solution as an efficacious single-step formulation may yield significant benefits for clinical cardiac surgery. It is likely that the film and solution play similar roles in the present study.

The mechanisms responsible for the effect of NOCC products on adhesion formation are not clear. Evidence suggests that fibroblast activation and deposition at the site of tissue injury are pivotal events in the initial stages of adhesion formation, and that the presence of a physical barrier between injured tissue surfaces during the critical stages of early healing (16-36 hours postsurgery) limits tissue apposition and minimizes fibrin matrix and adhesion formation. ${ }^{11-13} \mathrm{We}$ previously demonstrated that NOCC solution inhibits fibroblast activation and prevents fibroblast from adhering to NOCC-coated surfaces in vitro. Blocking the important deposition step could be the mechanism by which NOCC products prevent adhesion formation. It is possible that NOCC functions in vivo to block fibroblast attachment to the injured surfaces, thus interrupting fibrin matrix formation. In the case of the NOCC film, this is aided within the first few days by the presence of a direct physical barrier between the 2 injured surfaces, preventing the physical contact required for effective adhesion formation.

\section{CONCLUSIONS}

The use of physical barriers to prevent postsurgical adhesion formation has been demonstrated in many experimental models. ${ }^{3,14-17}$ An ideal physical barrier should not affect wound healing, not evoke fibrosis, be stable during the initial stages of adhesion formation but then degrade, and be efficacious in the presence of blood and body fluids. ${ }^{13,18}$ NOCC seems to possess all of these properties. We previously demonstrated that NOCC does not limit wound healing in both a rat skin healing model and a large bowel anastomosis model. ${ }^{7}$ Our in vivo evidence to date strongly suggests that NOCC does not stimulate fibrosis and rapidly degrades. ${ }^{10}$ The data from the experimentation presented confirm that NOCC is efficacious in reducing adhesion formation after cardiac surgery. Taken together, these data suggest that NOCC products satisfy the requirements for an ideal physical barrier to prevent postsurgical adhesions.

\section{References}

1. Macmanus Q, Okies JE, Phillips SJ, Starr A. Surgical considerations in patients undergoing repeat median sternotomy. J Thorac Cardiovasc Surg. 1975;69: 138-43.

2. Okuyama N, Wang CY, Rose EA, Rodgers KE, Pines E, diZerega GS, et al Reduction of retrosternal and pericardial adhesions with rapidly resorbable polymer films. Ann Thorac Surg. 1999;68:913-8.

3. Tsukihara H, Takamoto S, Kitahori K, Matsuda K, Murakami A, Novick RJ, et al. Prevention of postoperative pericardial adhesions with a novel regenerative collagen sheet. Ann Thorac Surg. 2006;81:650-7.

4. Chorny M, Mishaly D, Leibowitz A, Domb AJ, Golomb G. Site-specific delivery of dexamethasone from biodegradable implants reduces formation of pericardial adhesions in rabbits. $J$ Biomed Mater Res A. 2006;78:276-82.

5. Lu JH, Chang Y, Sung HW, Chiu YT, Yang PC, Hwang B. Heparinization on pericardial substitutes can reduce adhesion and epicardial inflammation in the dog. J Thorac Cardiovasc Surg. 1998;115:1111-20.

6. Kennedy R, Costain DJ, McAlister VC, Lee TD. Prevention of experimental postoperative peritoneal adhesions by N, O-carboxymethyl chitosan. Surgery. 1996 120:866-70.

7. Costain DJ, Kennedy R, Ciona C, McAlister VC, Lee TD. Prevention of postsurgical adhesions with $\mathrm{N}$, O-carboxymethyl chitosan: examination of the most efficacious preparation and the effect of N, O-carboxymethyl chitosan on postsurgical healing. Surgery. 1997;121:314-9.

8. Zhou J, Elson C, Lee TDG. Reduction in post surgical adhesion formation and reformation after abdominal surgery using N, O-carboxymethyl Chitosan. Surgery. 2004; 135:307-12.

9. Diamond MP, Luciano A, Johns DA, Dunn R, Young P, Bieber E. Reduction of postoperative adhesions by N, O-carboxymethylchitosan: a pilot study. Fertil Steril. 2003;631-6.

10. Zhou J, Liwski RS, Elson C, Lee TDG. Reduction in postsurgical adhesion formation after cardiac surgery in a rabbit model using N, O-carboxymethyl chitosan to block cell adherence. J Thorac Cardiovasc Surg. 2008;135:777-83.

11. Clark RAF. Wound Repair, Overview and General Considerations. The Molecular and Cellular Biology of Wound Repair. 2nd ed. New York: Plenum Press; 1996.

12. Singer II, Scott S, Kawka DW, Kazazis DM, Gailit J, Ruoslahti E. Cell surface distribution of fibronectin and vitronectin receptors depends on substrate composition and extracellular matrix accumulation. J Cell Biol. 1988;106:2171-82. 
13. Ricketts SA, Sibbons PD, Green CJ. Quantitative analysis of the development of experimentally induced post surgical adhesions: a microstereological study. Int $J$ Exp Pathol. 1999;80:325-34.

14. Harris ES, Morgan RF, Rodeheaver GT. Analysis of the kinetics of peritoneal adhesion formation in the rat and evaluation of potential antiadhesive agents. Surgery. 1995;117:663-9.

15. Miyamoto S, Katz BZ, Lafrenie RM, Yamada KM. Fibronectin and integrins in cell adhesion, signaling, and morphogenesis. Ann N Y Acad Sci. 1998;857: 119-29.
16. Sommerfeldt DW, McLeod KJ, Rubin CT, Hadjiargyrou M. Differential phosphorylation of paxillin in response to surface-bound serum proteins during early osteoblast adhesion. Biochem Biophys Res Commun. 2001;285:355-63.

17. Johns DB, Rodgers KE, Donahue WD, Kiorpes TC, diZerega GS. Reduction of adhesion formation by postoperative administration of ionically cross-linked hyaluronic acid. Fertil Steril. 1997;68:37-42.

18. Rodgers KE, Johns DB, Girgis W, Campeau J, diZerega GS. Reduction of adhesion formation with hyaluronic acid after peritoneal surgery in rabbits. Fertil Steril. 1997;67:553-8. 